\title{
HARMONIC MAPPINGS OF MULTIPLY CONNECTED DOMAINS
}

\author{
Peter Duren and Walter Hengartner
}

In this paper the theorem of Radó-Kneser-Choquet is extended in two different ways to multiply connected domains. One is a direct continuation of Kneser's idea and has nothing to do with convexity; while the other asserts that a finitely connected domain can be mapped harmonically with prescribed outer boundary correspondence onto a given convex domain with suitable punctures. It is also shown that a domain containing infinity admits a unique harmonic mapping, with standard normalization at infinity, onto a punctured plane. For domains of connectivity $n$ the dilatation of the canonical mapping covers the unit disk exactly $2 n$ times. Furthermore, no other normalized harmonic mapping has the same dilatation.

In 1926, T. Radó [22] posed the problem to show that for any homeomorphism of the unit circle onto the boundary $\partial \Omega$ of a bounded convex domain $\Omega$, the harmonic extension $f$ maps the unit disk $\mathbb{D}$ univalently onto $\Omega$. In response, H. Kneser [14] supplied an elegant proof. Some 20 years later G. Choquet [4], apparently unaware that the theorem was known, rediscovered it and gave another proof. Fortunately, the two proofs are different and even for simply connected domains they have different generalizations.

The dichotomy between the two approaches of Kneser and Choquet comes into sharper focus as the theorem is generalized to multiply connected domains. In presenting these generalizations, it will be expedient to distinguish between "Kneser's theorem" and "Choquet's theorem". Kneser's proof has little to do with convexity, while Choquet's proof uses convexity in a more essential way. Indeed, Kneser's proof applies (as he indicates in [14]) when $\Omega$ is not convex, under the additional hypothesis that $f(\mathbb{D}) \subset \Omega$. We shall see that the main idea of his proof carries over to multiply connected domains. On other hand, by methods more akin to Choquet's proof we will show that a finitely connected domain $D$ can be mapped harmonically, with prescribed boundary values, onto a given convex domain with punctures at suitable points. Another result is that $D$ can be mapped harmonically onto a punctured plane, and such a mapping is unique up to a normalization. Our proofs adapt an idea of Clunie and Sheil-Small [5], which gives yet another proof of the Radó-Kneser-Choquet theorem. 


\section{Introduction.}

Every complex-valued function $f$ harmonic in a domain $D \subset \mathbb{C}$ has a local representation $f=h+\bar{g}$, unique up to an additive constant, where $h$ and $g$ are analytic. Unless $D$ is simply connected, $h$ and $g$ need not extend globally to single-valued analytic functions. The Jacobian of the mapping $f$ is

$$
J=\left|f_{z}\right|^{2}-\left|f_{\bar{z}}\right|^{2}=\left|h^{\prime}\right|^{2}-\left|g^{\prime}\right|^{2},
$$

where $f_{z}=\frac{1}{2}\left(f_{x}-i f_{y}\right)$ and $f_{\bar{z}}=\frac{1}{2}\left(f_{x}+i f_{y}\right), z=x+i y$. If $f$ is locally univalent, a theorem of Lewy [18] says that $J(z) \neq 0$. The function $f$ is said to be orientation-preserving if $J(z)>0$ and orientation-reversing if $J(z)<0$. Note that $f$ preserves orientation if and only if its conjugate $\bar{f}$ reverses orientation.

If $f$ is an orientation-preserving harmonic function in $D$, then $f_{z}$ is analytic and nonvanishing in $D$; while the ratio $a=\overline{f_{\bar{z}}} / f_{z}=g^{\prime} / h^{\prime}$ is an analytic function satisfying $|a(z)|<1$. This function $a(z)$ is sometimes called the second complex dilatation of $f$. Note that the Jacobian can be expressed as $J=\left|h^{\prime}\right|^{2}\left(1-|a|^{2}\right)$. In the converse direction, it is known (see [11]) that if $a(z)$ is analytic and satisfies $|a(z)| \leq k<1$ in the unit disk $\mathbb{D}$, and if $\Omega$ is a prescribed simply connected domain bounded by an analytic Jordan curve, then there is a univalent orientation-preserving mapping $f$ of $\mathbb{D}$ onto $\Omega$ satisfying the Beltrami equation $\overline{f_{\bar{z}}}=a f_{z}$.

In simplest form, the theorem of Radó-Kneser-Choquet $[22, \mathbf{1 4}, 4]$ can be stated as follows. If $\Omega \subset \mathbb{C}$ is a bounded convex domain and if $f^{*}$ is an orientation-preserving homeomorphism of the unit circle $\mathbb{T}=\partial \mathbb{D}$ onto the boundary curve $\partial \Omega$, then its harmonic extension

$$
f(z)=\frac{1}{2 \pi} \int_{0}^{2 \pi} \frac{1-|z|^{2}}{\left|e^{i t}-z\right|^{2}} f^{*}\left(e^{i t}\right) d t
$$

maps $\mathbb{D}$ univalently onto $\Omega$. The theorem remains true, as Kneser's proof shows, if $\Omega$ is not convex but $f(\mathbb{D}) \subset \Omega$. Also, the boundary function $f^{*}$ need only be a "weak homeomorphism", possibly constant on some arcs. If $\Omega$ is convex, Choquet's proof applies at once to give the univalence even if $f^{*}$ has jump discontinuities (suitably restricted), but then the range of $f$ is typically a polygonal region inscribed in the boundary of $\Omega$.

In attempting to generalize the theorem to multiply connected domains, the first problem is to define the notion of an orientation-preserving homeomorphism of the boundary. If for instance the target region has an interior slit, it is not easy to specify the direction in which the image point "goes around" the corresponding boundary component. For a suitable definition it will be convenient to interpose certain conformal mappings. 
First consider a mapping $f^{*}\left(e^{i t}\right)=e^{i \theta(t)}$ of the unit circle onto itself. This mapping $f^{*}$ will be called an orientation-preserving weak homeomorphism if $\theta(t)$ is continuous and nondecreasing, and $\theta(2 \pi)=\theta(0)+2 \pi$. Thus as the point $e^{i t}$ runs once around the unit circle, its image $e^{i \theta}$ runs once around the circle, moving continuously in the same direction, although it may have intervals of constancy.

Now let $D$ be a bounded Jordan domain of finite connectivity $n$, with outer boundary $C_{0}$ and inner boundary curves $C_{1}, C_{2}, \ldots, C_{n}$. Let $D_{0}$ denote the bounded component of $\mathbb{C} \backslash C_{0}$ (this is $D$ with the "holes filled in"). For $j=1,2, \ldots, n$, let $K_{j}$ be the bounded component of $\mathbb{C} \backslash \Omega$ that contains $C_{j}$. Let $\Omega$ be another bounded domain of the same connectivity $n$, whose outer boundary $\Gamma_{0}$ is a Jordan curve and whose inner boundary components $\Gamma_{j}$ (for $j=1,2, \ldots, n$ ) are locally connected. For $1 \leq j \leq n$, let $\Delta_{j}$ denote the component of $\mathbb{C} \backslash \Omega$ that contains $\Gamma_{j}$. Let $\varphi_{0}$ be a conformal mapping of the unit disk $\mathbb{D}$ onto $D_{0}$, and let $\psi_{0}$ map $\mathbb{D}$ conformally onto $\Omega_{0}$. Finally, let $\varphi_{j}$ (resp. $\psi_{j}$ ) map the exterior of $\mathbb{D}$ onto the complement of $K_{j}$ (resp. $\Delta_{j}$, whenever $\Delta_{j}$ is not a single point), with $\varphi_{j}(\infty)=\psi_{j}(\infty)=\infty$. Since the boundary components of $\Omega$ are locally connected, the mappings $\varphi_{j}$ and $\psi_{j}$ have continuous extensions to the boundary. A mapping $f^{*}$ from $\partial \Omega$ is said to be an orientation-preserving weak homeomorphism if $f^{*}\left(C_{j}\right)=\Gamma_{j}$ for $0 \leq j \leq n$ and the composition $\psi_{j}^{-1} \circ f^{*} \circ \varphi_{j}$ is an orientation-preserving weak homeomorphism of the unit circle onto itself unless $\Delta_{j}$ is a single point (and hence $f^{*}$ is constant on $C_{j}$ ).

\section{Generalization of Kneser's theorem.}

We are now prepared to state a generalization of Kneser's theorem to multiply connected domains.

Theorem 1. Let $D$ be a bounded finitely connected Jordan domain, and let $\Omega$ be a bounded domain of equal connectivity whose outer boundary is a Jordan curve and whose inner boundary components are locally connected. Let $f^{*}$ be an orientation-preserving weak homeomorphism of $\partial D$ onto $\partial \Omega$. Let $f$ be the solution of the Dirichlet problem, the harmonic extension of $f^{*}$ to $D$. If $f(D) \subset \Omega$, then $f$ maps $D$ univalently onto $\Omega$. Conversely, if $f$ is univalent in $D$, then $f(D)=\Omega$.

Since the harmonic extension $f$ is in particular a continuous function on $\bar{D}$ that maps $\partial D$ onto $\partial \Omega$, a known theorem from topological degree theory (see for instance [24]) guarantees that $f(D) \supset \Omega$. Thus the hypothesis $f(D) \subset \Omega$ implies $f(D)=\Omega$, and an equivalent formulation of the theorem is that $f$ maps $D$ univalently onto $\Omega$ if and only if $f(D)=\Omega$. 
The basic strategy of proof is that of Kneser [14], but the presence of additional boundary components creates an additional complication, as will be seen below. In the spring of 1994 we used a connectedness argument to overcome the problem, and we announced the result in [6] and [3]. However, we later received a preprint from Lyzzaik [19] which suggested to us an idea of homotopy to handle the difficulty in a more elegant way. In the following proof we have modified our original argument accordingly.

Proof of theorem. The first step is to show that $f$ is locally univalent in $D$, or equivalently that its Jacobian $J(z) \neq 0$ in $D$. The second step is then to apply the argument principle for harmonic functions to conclude that $f$ is globally univalent. The third step is to show that $f$ cannot be univalent in $D$ if it assumes a value outside $\Omega$.

Step 1. Suppose on the contrary that $J\left(z_{0}\right)=0$ for some point $z_{0}$ in $D$. Then with the notation $f(z)=u(z)+i v(z)$, the system of linear equations

$$
\alpha u_{x}\left(z_{0}\right)+\beta v_{x}\left(z_{0}\right)=0, \quad \alpha u_{y}\left(z_{0}\right)+\beta v_{y}\left(z_{0}\right)=0
$$

has a nontrivial real solution $(\alpha, \beta)$. In other words, the real-valued harmonic function $\psi(z)=\alpha u(z)+\beta v(z)$ has a critical point at $z$. Since $f(D)$ does not lie on a line, $\psi(z)$ cannot be constant on $D$. Let $c=\psi\left(z_{0}\right)$ and let $\Lambda$ denote the component of the level-set $\psi(z)=c$ that contains $z_{0}$. At the critical point, $\Lambda$ splits into $2 m$ branches $(m \geq 2)$ emanating from $z_{0}$ at equal angles. As $\Lambda$ extends away from $z_{0}$ it may branch again as it meets other critical points, but it cannot terminate at any interior point of $D$.

Let $\mathcal{L}$ be the line in the $w$-plane $(w=u+i v)$ given by the equation $\alpha u+\beta v=c$, and let $L$ be the component of $\mathcal{L} \cap \Omega$ containing the point $w_{0}=f\left(z_{0}\right)$. Thus $L$ is an open segment in $\Omega$ with endpoints $P$ and $Q$ on $\partial \Omega$. Since $f(D) \subset \Omega$, it is clear that $f(\Lambda) \subset L$.

In what follows, we will say that a set $A \subset \mathbb{C}$ clusters at a set $B$ if there is a sequence of points $z_{k}$ in $A$ which converges to a point of $B$. Note that $f^{-1}(P)$ and $f^{-1}(Q)$ are disjoint intervals of $\partial D$, possibly single points, since $f^{*}$ is a weak homeomorphism. (Here $f^{-1}(P)$ and $f^{-1}(Q)$ are the prime ends determined by the segment $L$.) If the set $\Lambda$ clusters at $\partial D$, it must do so either on $f^{-1}(P)$ or on $f^{-1}(Q)$. If $\Lambda$ does not cluster at $\partial D$, it must contain a closed loop in $D$. Because $\Lambda$ has at least 4 branches emanating from $z_{0}$, there are two possibilities (not mutually exclusive) to consider. Either

(i) $\Lambda$ contains a closed loop in $D$; or

(ii) Two different branches of $\Lambda$ cluster at the same set $f^{-1}(P)$ or $f^{-1}(Q)$.

Suppose first that (i) occurs, so that $\Lambda$ contains a Jordan curve $\hat{\Lambda}$ in $D$ on which $\psi(z) \equiv c$. If $\hat{\Lambda}$ surrounds no boundary components of $D$, then since $\psi$ is harmonic inside $\hat{\Lambda}$ it follows from the maximum principle that $\psi$ 
is constant there and so $\psi(z) \equiv c$ in $D$, which is certainly not true because $f(D)$ is not contained in a line. Thus we may suppose that $\hat{\Lambda}$ surrounds some interior boundary component, say $C_{1}$. However, we will show that this too is impossible. Extend the boundary function $f^{*}$ to a continuous function $F_{j}$ on $K_{j}$ such that $F_{j}\left(K_{j}\right)=\Lambda_{j}$ for $j=2, \ldots, n$. (See Section 1 for the notation.) Extend the given harmonic function $f$ to a continuous function $F$ on the doubly connected domain $D_{1}=D \cup K_{2} \cup \cdots \cup K_{n}$ by defining $F=F_{j}$ on $K_{j}$ for $j=2, \ldots, n$. Recall now that by hypothesis, $f(D)=\Omega$. Thus $F$ is a continuous mapping of $D_{1}$ onto the doubly connected domain $\Omega_{1}=\Omega \cup \Delta_{2} \cup \cdots \cup \Delta_{n}$. Moreover, $\hat{\Lambda}$ is homotopic to $C_{0}$ in $D_{1}$, while its image $\hat{L}=F(\hat{\Lambda}) \subset L$ lies on a line segment in $\Omega \subset \Omega_{1}$ (since $f(D) \subset \Omega$ ), and so $\hat{L}$ is not homotopic to $\Gamma_{0}=f^{*}\left(C_{0}\right)$ in $\Omega_{1}$. But this is impossible, because homotopy classes are invariant under continuous mappings. This shows that $\Lambda$ cannot have the property (i); it cannot contain a closed loop in $D$. A similar argument rules out the possibility (ii). The assumption $J\left(z_{0}\right)=0$ has therefore led to a contradiction, and we conclude that $J(z) \neq 0$ in $D$.

Step 2. Since $f$ is locally univalent and its boundary function $f^{*}$ is orientation-preserving, it follows that $f$ is an orientation-preserving harmonic function, whose dilatation $a$ satisfies $|a(z)|<1$ in $D$. A standard appeal to the argument principle for harmonic functions (see [7], for instance) now shows that $f$ maps $D$ univalently onto $\Omega$.

Step 3. Suppose now that $f$ is univalent in $D$ but $f\left(z_{1}\right) \notin \Omega$ for some point $z_{1} \in D$. By Lewy's theorem $f$ is orientation-preserving in $D$ and its dilatation $a=\overline{f_{\bar{z}}} / f_{z}$ satisfies $|a(z)|<1$. In particular, $f$ is an open mapping. Choose a point $z_{0} \in D$ such that $f\left(z_{0}\right) \in \Omega$, and let $z=z(t), 0 \leq t \leq 1$, be a Jordan arc in $D$ from $z_{0}$ to $z_{1}$. Define

$$
\tau=\inf \{t \in[0,1]: z(t) \notin \Omega\},
$$

and let $z_{2}=z(\tau)$. Then $w_{2}=f\left(z_{2}\right) \in \partial \Omega$, and $f$ maps each open neighborhood of $z_{2}$ onto an open neighborhood of $w_{2}$, since $f$ is an open mapping. However, $f$ has a continuous extension to the boundary, and $f(\zeta)=w_{2}$ for some point $\zeta \in \partial D$. From this we conclude that $f$ assumes certain values near $w_{2}$ at two distinct points in $D$, one near $z_{2}$ and the other near $\zeta$. In other words, $f$ is not univalent. Thus $f(D) \subset \Omega$ if $f$ is univalent in $D$. But we have already noted that $f(D) \supset \Omega$ for any continuous extension of the boundary function $f^{*}$, so $f(D)=\Omega$ if $f$ is univalent. This concludes the proof of Theorem 1 .

The key to Kneser's proof is the observation that wherever the Jacobian of a complex-valued harmonic function $f=u+i v$ vanishes, some real-valued 
harmonic function $\psi=\alpha u+\beta v$ has a critical point. If the Jacobian matrix has rank one at this point $z_{0}$, or equivalently if $\left|h^{\prime}\left(z_{0}\right)\right|=\left|g^{\prime}\left(z_{0}\right)\right| \neq 0$, where $f=h+\bar{g}$ is a local representation of $f$ in terms of analytic functions, then there is exactly one line segment passing through $w_{0}=f\left(z_{0}\right)$ whose preimage splits at $z_{0}$. Surprisingly, the direction of this line depends only on the dilatation of $f$ at $z_{0}$; specifically, the direction is $\overline{a\left(z_{0}\right)}{ }^{1 / 2}$. The following proposition can be verified by straightforward calculation.

Proposition. Let $f=h+\bar{g}$ be harmonic in a domain D, and suppose that $f(D)$ does not lie on a line. Suppose that the Jacobian $J=\left|h^{\prime}\right|^{2}-\left|g^{\prime}\right|^{2}$ vanishes at some point $z_{0}$ in $D$, with $\left|h^{\prime}\left(z_{0}\right)\right|=\left|g^{\prime}\left(z_{0}\right)\right| \neq 0$. Let $\Lambda$ be the set of points $z$ in $D$ for which $f(z)=u(z)+i v(z)$ lies on a line $\alpha u+\beta v=c$ passing through $w_{0}=f\left(z_{0}\right)$. Then $\Lambda$ splits at $z_{0}$ if and only if $\alpha / \beta=\tan \varphi$, where $a\left(z_{0}\right)=g^{\prime}\left(z_{0}\right) / h^{\prime}\left(z_{0}\right)=e^{2 i \varphi}$ is the dilatation of $f$ at $z_{0}$.

\section{Generalization of Choquet's theorem.}

Suppose now that the Jordan domain $D$ is simply connected and that $\Omega$ is a bounded convex domain. Let $f^{*}$ be an orientation-preserving weak homeomorphism of $\partial D$ onto $\partial \Omega$. Then according to Choquet's theorem, the harmonic extension $f$ maps $D$ univalently onto $\Omega$. This result is contained in Kneser's earlier theorem, since the convexity of $\Omega$ ensures that $f(D) \subset \Omega$, but we call it Choquet's theorem because his proof made stronger use of convexity. We shall now generalize Choquet's theorem to multiply connected domains, where the convexity hypothesis will be essential.

Theorem 2. Let $D$ be a finitely connected domain bounded by Jordan curves $C_{0}, C_{1}, \ldots, C_{n}$, where $C_{0}$ is the outer boundary component. Let $\Omega$ be a bounded convex domain. Suppose that $f^{*}$ is an orientation-preserving weak homeomorphism of $C_{0}$ onto $\partial \Omega$. Then there is a function $f$ harmonic in $D$ and continuous in $\bar{D}$, mapping $D$ univalently onto $\Omega$ with $n$ points removed, with the prescribed boundary values: $f(z)=f^{*}(z)$ on $C_{0}$.

It must be emphasized that the locations of the punctures are not prescribed independently, but will depend upon the given boundary function $f^{*}$. The theorem was previously known for doubly connected domains $(n=1)$. Nitsche [21] constructed a harmonic mapping of an annulus onto a disk punctured at the center. Bshouty and Hengartner [2] proved Theorem 3 for doubly connected domains, where $D$ may be taken to be an annulus, and they found in that case that the inner boundary component may always be mapped to the average value of $f^{*}$. 
Proof of theorem. Applying an appropriate conformal premapping, we may assume without loss of generality that each boundary component of $D$ is an analytic Jordan curve. We first solve the Dirichlet problem to produce a function $F$ harmonic in $D$ and continuous in $\bar{D}$, with $F(z)=f^{*}(z)$ on $C_{0}$ and $F(z) \equiv 0$ on the rest of the boundary. Evidently $F$ need not be univalent, and can not be if $n \geq 2$, but it has the other required properties.

Let $\omega_{j}$ be the harmonic measure of $C_{j}$ with respect to $D, j=0,1, \ldots, n$. Let

$$
p_{j k}=\int_{C_{k}} \partial \omega_{j} / \partial n d s, \quad j, k=0,1, \ldots, n,
$$

denote the period of the harmonic conjugate of $\omega_{j}$ around $C_{k}$, and let

$$
\gamma_{k}=\int_{C_{k}} \partial F / \partial n d s, \quad k=1, \ldots, n
$$

It is well known (see e.g. Nehari [20], Chapter 1) that the submatrix $\left(p_{j k}\right)$ for $j, k=1, \ldots, n$ is nonsingular. Thus the linear system

$$
\sum_{j=1}^{n} \lambda_{j} p_{j k}+\gamma_{k}=0, \quad k=1, \ldots, n,
$$

has a unique complex solution $\left(\lambda_{j}\right)$. Define

$$
f=F+\sum_{j=1}^{n} \lambda_{j} \omega_{j}
$$

Then $f$ is a harmonic function with boundary values $f(z)=f^{*}(z)$ on $C_{0}$ and $f(z) \equiv \lambda_{k}$ on $C_{k}$ for $k=1, \ldots, n$. By construction,

$$
\int_{C_{k}} \partial f / \partial n d s=0, \quad k=0,1, \ldots, n .
$$

We claim now that all of the points $\lambda_{k}$ lie in $\Omega$. If not, then for a suitable choice of angle $\alpha$,

$$
\max _{z \in \bar{D}} \operatorname{Re}\left\{e^{i \alpha} f(z)\right\}=\operatorname{Re}\left\{e^{i \alpha} \lambda_{j}\right\}
$$

for some $\lambda_{j}$. This conclusion is justified by the maximum principle for harmonic functions and the convexity of $\Omega$, together with the properties $f\left(C_{0}\right)=$ $\partial \Omega$ and $f\left(C_{k}\right)=\lambda_{k}$ for $k=1, \ldots, n$. Preceding $f$ by a conformal mapping, we may suppose that $C_{j}$ is a circle $|z-\zeta|=\rho$ with center $\zeta$ and radius $\rho$. By conformal invariance,

$$
r \frac{\partial}{\partial r} \int_{0}^{2 \pi} f\left(\zeta+r e^{i t}\right) d t=\int_{C_{j}} \frac{\partial f}{\partial n} d s=0 .
$$


Thus

$$
\frac{1}{2 \pi} \int_{0}^{2 \pi} f\left(\zeta+r e^{i t}\right) d t \equiv \lambda_{j} \quad \rho<r<\rho+\varepsilon,
$$

for some $\varepsilon>0$. It now follows from the extremal property that $\operatorname{Re}\left\{e^{i \alpha} f(z)\right\}=$ $\operatorname{Re}\left\{e^{i \alpha} \lambda_{j}\right\}$ in the annulus $\rho<|z|<\rho+\varepsilon$ and so (by harmonic continuation) throughout the domain $D$. But this is not possible, since $f$ maps $C_{0}$ onto $\partial \Omega$ and so its values in $D$ do not lie on a line. Thus we conclude that all $\lambda_{k}$ are in $D$.

It remains to show that $f$ is univalent in $D$. First observe that $f$ has a global representation $f=h+\bar{g}$, where $h$ and $g$ are analytic in $D$. Indeed, the property

$$
\int_{C_{k}} \frac{\partial f}{\partial n} d s=0, \quad k=0,1, \ldots, n,
$$

says that $f$ has a single-valued conjugate function in $D$. But if $f=h+\bar{g}$ is a local representation, then $\operatorname{Re}\{f\}=\operatorname{Re}\{h+g\}$ and $\operatorname{Im}\{f\}=\operatorname{Im}\{h-g\}$. It follows that $h+g$ and $h-g$ have single-valued analytic extensions to $D$. Thus $h$ and $g$ extend to single-valued analytic functions in $D$, and $f$ has a global representation $f=h+\bar{g}$.

Now define the analytic function

$$
\phi_{\alpha}=e^{i \alpha} h-e^{-i \alpha} g=e^{i \alpha} f-2 \operatorname{Re}\left\{e^{-i \alpha} g\right\},
$$

where $\alpha$ is a real parameter. Since the boundary components $C_{j}$ are analytic curves and $f$ is constant on $C_{j}(j=1, \ldots, n)$, we see that $f$ admits a harmonic continuation and thus $h$ and $g$ admit analytic extensions across $C_{j}$. Thus the image sets $\phi_{\alpha}\left(C_{j}\right)$ are bounded horizontal intervals, $j=1, \ldots, n$. Because $f\left(C_{0}\right)=\partial \Omega$ bounds a convex region, it follows that $\operatorname{Im}\left\{\phi_{\alpha}\right\}$ is nondecreasing on one arc of $C_{0}$ and nonincreasing on the complementary arc. Since $\phi_{\alpha}$ is an open mapping, this implies that $\phi_{\alpha}\left(C_{0}\right)$ is a curve in the extended complex plane bounding a domain convex in the horizontal direction, containing all of the segments $\phi_{\alpha}\left(C_{j}\right), j=1, \ldots, n$. Let $G$ be the domain inside $\phi_{\alpha}\left(C_{0}\right)$ with the segments $\phi_{\alpha}\left(C_{j}\right)$ removed.

To see that $\phi_{\alpha}$ is univalent in $D$ and maps it onto $G$, we apply the argument principle. Fix a point $w_{0} \notin \partial G$ and note that

$$
\begin{aligned}
\int_{\partial D} d \arg \left\{\phi_{\alpha}(z)-w_{0}\right\} & =\int_{C_{0}} d \arg \left\{\phi_{\alpha}(z)-w_{0}\right\} \\
& = \begin{cases}2 \pi & \text { if } w_{0} \in G \\
0 & \text { if } w_{0} \notin G .\end{cases}
\end{aligned}
$$


Thus $\phi_{\alpha}$ is univalent in $D$, and it follows that

$$
\phi_{\alpha}^{\prime}(z)=e^{i \alpha} h^{\prime}(z)-e^{-i \alpha} g^{\prime}(z) \neq 0
$$

in $D$ for every $\alpha \in \mathbb{R}$. In particular, $\left|h^{\prime}(z)\right|+\left|g^{\prime}(z)\right| \neq 0$ and $|a(z)| \neq 1$ in $D$. Since $f^{*}$ is an orientation-preserving weak homeomorphism of $\partial D$ onto $\partial \Omega$, we conclude that $|a(z)|<1$ in $D$. This allows us to apply the argument principle [7] to $f$. For $w_{0} \notin \partial \Omega$ and $w_{0} \neq \lambda_{j}$ for $j=1, \ldots, n$, we see that

$$
\begin{aligned}
\int_{\partial D} d \arg \left\{f(z)-w_{0}\right\} & =\int_{C_{0}} d \arg \left\{f^{*}(z)-w_{0}\right\} \\
& = \begin{cases}2 \pi & \text { if } w_{0} \in \Omega \\
0 & \text { if } w_{0} \notin \Omega .\end{cases}
\end{aligned}
$$

Hence we conclude that $f$ is univalent in $D$ and maps it onto $\Omega$ with the points $\lambda_{k}$ removed. Since $f$ preserves the connectivity of $D$, the $n$ points $\lambda_{k}$ must all be distinct. This concludes the proof of Theorem 3 .

As corollaries of the proof, we can state two closely related results. The first may be viewed as a direct generalization of Choquet's theorem, because it says that any harmonic extension of the prescribed boundary function is univalent if it is constant on the inner boundary components, under a mild hypothesis that holds automatically for simply connected domains. The second corollary generalizes a characterization of convex mappings due to Clunie and Sheil-Small [5].

Corollary 1. Let $D$ be a finitely connected domain bounded by Jordan curves $C_{0}, C_{1}, \ldots, C_{n}$, where $C_{0}$ is the outer boundary component. Let $\Omega$ be a bounded convex domain. Suppose that $f^{*}$ is an orientation-preserving weak homeomorphism of $C_{0}$ onto $\partial \Omega$. Let $f=h+\bar{g}$ be any function harmonic in $D$ and continuous in $\bar{D}$, with $h$ and $g$ (globally) analytic in $D$, such that $f(z)=f^{*}(z)$ on $C_{0}$ and $f(z)$ is constant on each of the inner boundary components $C_{1}, \ldots, C_{n}$. Then $f$ is a univalent mapping of $D$ onto $\Omega$ minus the points $f\left(C_{k}\right), k=1, \ldots, n$.

Corollary 2. Let $f=h+\bar{g}$ be harmonic in a finitely connected domain $D$ and continuous in $\bar{D}$. Then $f$ is a univalent mapping of $D$ onto a punctured convex domain if and only if for each $\alpha \in \mathbb{R}$ the function $\phi_{\alpha}=e^{i \alpha} h-e^{-i \alpha} g$ is a conformal mapping of $D$ onto a slit domain convex in the horizontal direction.

This last result will be further generalized in Section 4. We now conclude the present section with some remarks and two examples. First we note 
that Choquet's theorem fails for unbounded convex domains. An example was given in [2], but the following example is more instructive. Begin with the conformal mapping $\zeta=\xi+i \eta=2 z /(1+z)$ of the unit disk $|z|<1$ onto the half-plane $\operatorname{Re}\{\zeta\}<1$. Note that the harmonic polynomial

$$
p(\zeta)=\frac{1}{2}(\zeta+\bar{\zeta})+\frac{1}{4}\left(\zeta^{2}-\bar{\zeta}^{2}\right)=\xi(1+i \eta)
$$

maps each vertical line $\xi=\xi_{0} \neq 0$ univalently onto itself, while it sends the whole line $\xi=0$ to the origin. Thus

$$
w=f(z)=p\left(\frac{2 z}{1+z}\right)
$$

maps the unit circle univalently onto the line $\operatorname{Re}\{w\}=1$, but its harmonic extension to the disk is not univalent.

Choquet's theorem also fails in spaces of higher dimension. Laugesen [17] has constructed a homeomorphism of the unit sphere in $\mathbb{R}^{3}$ whose harmonic extension to the ball is not univalent.

Example 1. As we have already mentioned, one cannot prescribe the locations of the punctures in Theorem 3 or in Corollary 1 . In the special case where $n=1$, we may take $D$ to be an annulus $\rho<|z|<1$, so that $C_{1}$ is the circle $|z|=\rho$. Then the construction in the proof of Theorem 2 (or in [2]) gives

$$
\int_{C_{1}} \frac{\partial f}{\partial n} d s=0
$$

so that $\int_{0}^{2 \pi} f\left(r e^{i \theta}\right) d \theta$ is constant for $\rho<r<1$ and the puncture is

$$
\lambda_{1}=\frac{1}{2 \pi} \int_{0}^{2 \pi} f^{*}\left(e^{i \theta}\right) d \theta
$$

the average of the prescribed boundary function. However, there are other choices for $\lambda_{1}$. For instance, take $\Omega$ to be the unit disk and $f^{*}\left(e^{i \theta}\right)=e^{i \theta}$. Then $\int_{0}^{2 \pi} f^{*}\left(e^{i \theta}\right) d \theta=0$ and $\lambda_{1}=0$ is a possible choice. Here the solution of the Dirichlet problem is

$$
f(z)=\frac{z-\rho^{2} / \bar{z}}{1-\rho^{2}}
$$

which maps $D$ univalently onto $\Omega \backslash\{0\}$. But for any complex constant $c$ the function

$$
F(z)=\frac{z-\rho^{2} / \bar{z}}{1-\rho^{2}}+2 c \log |z|
$$

is also harmonic in $D$ and has the same boundary function $F\left(e^{i \theta}\right)=e^{i \theta}$. If $|c|<\rho /\left(1-\rho^{2}\right)$, we claim that $F$ maps $D$ univalently onto the disk punctured 
at $p_{1}=2 c \log \rho$. This will follow at once from the argument principle if we can show that the dilatation $A=\overline{F_{\bar{z}}} / F_{z}$ satisfies $|A(z)|<1$ in $D$. But direct calculation of the derivatives $F_{z}$ and $F_{\bar{z}}$ leads after some manipulation to the formula

$$
A(z)=\zeta \frac{\zeta+\bar{b}}{1+b \zeta}, \quad \zeta=\rho / z, b=c\left(1-\rho^{2}\right) / \rho
$$

Since $\rho<|\zeta|<1$ for $\rho<|z|<1$, it follows that $|A(z)|<1$ in $D$ if $|b|<1$, or if $|c|<\rho /\left(1-\rho^{2}\right)$. It is easily checked that $|2 c \log \rho|<1$ under the same condition.

Example 2. It is natural to ask whether the generalized version of Choquet's theorem (Corollary 1) still holds if $f$ is allowed to carry each inner boundary component to a horizontal segment, not necessarily a single point. If the dilatation of $f$ has the property $|a(z)|<1$, then the answer is YES, by the argument principle. But in general the answer is NO, as the following example shows.

Again let $n=1$ and let $D$ be the annulus $\rho<|z|<1$. Consider the harmonic function

$$
f(z)=4 z-\bar{z} / 3-1 /(6 z)-2 / \bar{z}
$$

or

$$
f\left(r e^{i \theta}\right)=\left(\frac{11 r}{3}-\frac{13}{6 r}\right) \cos \theta+i\left(\frac{13 r}{3}-\frac{11}{6 r}\right) \sin \theta .
$$

Then the mapping

$$
w=f\left(e^{i \theta}\right)=\frac{3}{2} \cos \theta+\frac{5}{2} i \sin \theta
$$

is an orientation-preserving homeomorphism from the unit circle onto the ellipse

$$
\left(\frac{2 u}{3}\right)^{2}+\left(\frac{2 v}{5}\right)^{2}=1, \quad w=u+i v
$$

Now choose $\rho=\sqrt{11 / 26}$, so that $\frac{13 \rho}{3}-\frac{11}{6 \rho}=0$ and $f$ maps the inner boundary circle $C_{1}=\{z:|z|=\rho\}$ onto a segment of the real axis. Specifically,

$$
f\left(C_{1}\right)=[-16 / \sqrt{286}, 16 / \sqrt{286}] \approx[-0.9461,0.9461],
$$

which lies inside the domain $\Omega$ bounded by the ellipse specified above. 
However, a simple calculation shows that $f$ has dilatation

$$
a(z)=\frac{2\left(6-z^{2}\right)}{24 z^{2}+1}
$$

and it is easy to check that $a( \pm i r)=-1$ for $r=\sigma=\sqrt{13 / 22}$, which satisfies $\rho<\sigma<1$. Thus the Jacobian of $f$ vanishes at points in $D$, so by Lewy's theorem $f$ cannot be univalent.

It is interesting to go a step farther and investigate the geometric behavior of this harmonic function $f$ in the annulus. Observe first that $f$ maps the circle $|z|=\sigma$ onto the vertical segment $i[-16 / \sqrt{286}, 16 / \sqrt{286}]$. The image of every other circle $|z|=r$, for $\rho<r<1$, is an ellipse. As $r$ decreases from 1 to $\sigma$, these ellipses shrink to the given vertical segment, and indeed $f$ maps the annulus $\sigma<r<1$ univalently onto $\Omega$ minus this vertical slit. However, as $r$ decreases from $\sigma$ to $\rho$, the ellipses begin to overlap and gradually flatten out to the given horizontal segment as folding occurs. Figure 1 shows the image of circles $|z|=r$ for $r$ in each of the intervals $\sigma<r<1$ and $\rho<r<\sigma$.

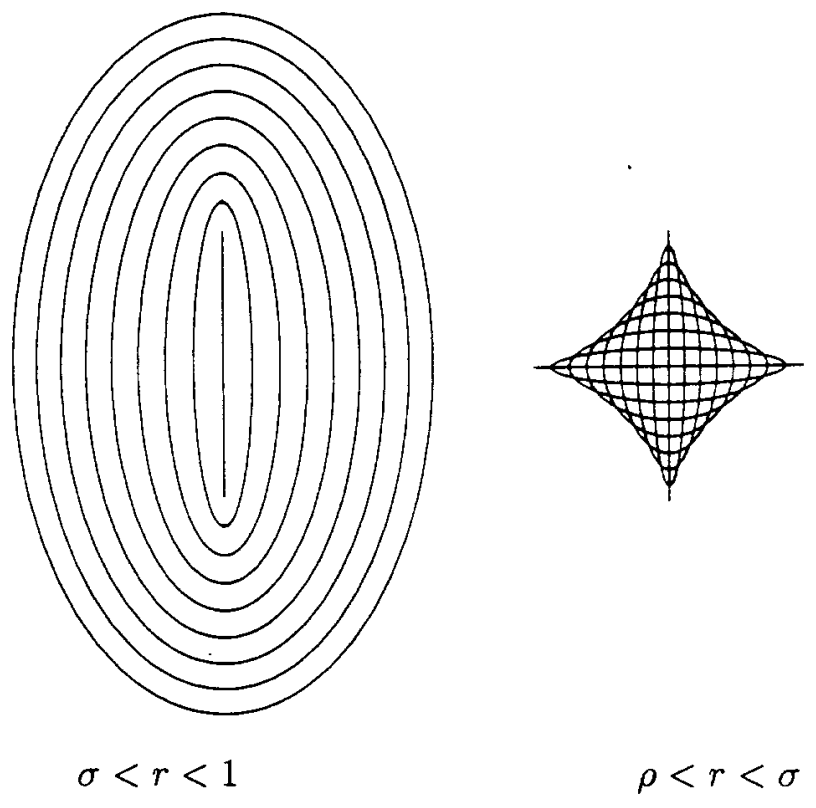

Figure 1.

\section{Harmonic mappings onto punctured planes.}

In this section we show that any domain can be mapped harmonically onto a punctured plane. It will be convenient to consider a domain in the extended 
complex plane $\hat{\mathbb{C}}$, containing the point at infinity. The simplest example is the domain $\Delta$ which lies outside the closed unit disk. It is easy to see that

$$
f(z)=z-1 / \bar{z}=(r-1 / r) e^{i \theta}, \quad z=r e^{i \theta},
$$

maps $\Delta$ univalently onto $\hat{\mathbb{C}} \backslash\{0\}$, the extended plane punctured at the origin. More generally, it was shown in [12] that a function $f$ provides a univalent orientation-preserving harmonic mapping of $\Delta$ onto a punctured sphere $\hat{\mathbb{C}} \backslash$ $\{p\}$ if and only if it has the form

$$
f(z)=p+b[z+c d \bar{z}+2(c+d) \log |z|-c d / z-1 / \bar{z}]
$$

for complex constants $b, c$ and $d$ with $b \neq 0,|c|<1$ and $|d| \leq 1$. The corresponding dilatation function is

$$
a(z)=(\bar{b} / b) \frac{1+\bar{c} z}{z+c} \frac{1+\bar{d} z}{z+d} .
$$

If we add the requirement that $f(z)=z+o(1)$ near infinity, then $p=0$, $b=1$ and $c=d=0$; so the function $f(z)=z-1 / \bar{z}$ is uniquely determined.

Given any domain $D \subset \hat{\mathbb{C}}$ containing the point at infinity, we will say that $f$ is a canonical harmonic punctured-plane mapping if $f$ provides a univalent harmonic mapping of $D$ onto some domain $\Omega$ of the form

$$
\Omega=\hat{\mathbb{C}} \backslash \bigcup_{j \in J}\left\{p_{j}\right\},
$$

for some points $p_{j} \in \mathbb{C}$; and if $f(z)=z+o(1)$ near infinity. Observe that the univalence of $f$ and the normalization at infinity imply that $f$ is orientationpreserving.

Theorem 3. Every domain $D \subset \hat{\mathbb{C}}$ containing the point at infinity admits a canonical harmonic punctured-plane mapping $F=H+\bar{G}$, where $H$ and $G$ are analytic (and single-valued) in $D$. If $\partial D$ has countably many components, such a mapping is unique.

Proof. Let $\left\{D_{n}\right\}$ be an exhaustion of $D$ by precompact domains $D_{n} \subset D$ of finite connectivity which contain the point at infinity and whose boundary components are analytic Jordan curves. Denote by $J_{\theta, n}$ the canonical conformal mapping of $D_{n}$ onto a parallel slit domain of inclination $\theta$ (with respect to the positive real axis), normalized at infinity by $J_{\theta, n}(z)=z+o(1)$. (For the existence and uniqueness of this mapping, and special properties, see $[\mathbf{1}, \mathbf{8}, \mathbf{9}]$.) In particular, $J_{0, n}\left(\right.$ resp.,$\left.J_{\pi / 2, n}\right)$ maps $D_{n}$ onto a horizontal (resp., vertical) slit domain, and

$$
J_{\theta, n}(z)=e^{i \theta}\left[\cos \theta J_{0, n}(z)-i \sin \theta J_{\pi / 2, n}(z)\right] .
$$


It is shown in [1] that $J_{\theta, n}(z)$ converges locally uniformly to a function $J_{\theta}(z)$ which maps $D$ conformally onto a parallel slit domain of inclination $\theta$ and is again normalized at infinity by $J_{\theta}(z)=z+o(1)$. If $\partial D$ has countably many components, then $J_{\theta}$ is the only function with these properties. Moreover, the identity

$$
J_{\theta}(z)=e^{i \theta}\left[\cos \theta J_{0}(z)-i \sin \theta J_{\pi / 2}(z)\right]
$$

remains valid.

Now define the function $F(z)=\operatorname{Re}\left\{J_{\pi / 2}(z)\right\}+\operatorname{Im}\left\{J_{0}(z)\right\}$. Observe that $F$ is harmonic in $D$, and it maps each component of $\partial D$ onto a single point. It also has the standard normalization $F(z)=z+o(1)$ near infinity. Finally, $F$ has a global decomposition $F=H+\bar{G}$, where

$$
H(z)=\frac{1}{2}\left[J_{\pi / 2}(z)+J_{0}(z)\right]
$$

and

$$
G(z)=\frac{1}{2}\left[J_{\pi / 2}(z)-J_{0}(z)\right]
$$

are single-valued analytic functions in $D$.

Now consider again the analytic function

$$
\Phi_{\alpha}=e^{i \alpha} H-e^{-i \alpha} G=e^{i \alpha} F-2 \operatorname{Re}\left\{e^{-i \alpha} G\right\},
$$

where $\alpha$ is a real parameter. The formulas for $H$ and $G$ show that $\Phi_{\alpha}$ takes the remarkable form

$$
\Phi_{\alpha}(z)=\cos \alpha J_{0}(z)+i \sin \alpha J_{\pi / 2}(z)=e^{i \alpha} J_{-\alpha}(z) .
$$

In particular, $\Phi_{\alpha}$ is univalent and hence $\Phi_{\alpha}^{\prime}(z) \neq 0$ in $D$ for each $\alpha \in \mathbb{R}$. By the definition of $\Phi_{\alpha}$, it follows that $\left|H^{\prime}(z)\right|+\left|G^{\prime}(z)\right| \neq 0$ and $|A(z)| \neq 1$ in $D$, where $A=G^{\prime} / H^{\prime}$ is the dilatation function of $F$. But the normalizations of $J_{0}$ and $J_{\pi / 2}$ at infinity show that $H^{\prime}(\infty)=1$ and $G^{\prime}(\infty)=0$, so $A(\infty)=0$ and we conclude that $|A(z)|<1$ in $D$.

We claim now that the composed function $Q=F \circ J_{0}^{-1}$ is univalent on the horizontal slit-domain $J_{0}(D)$. To see this, write $w=u+i v=Q(\zeta)$, where $\zeta=\xi+i \eta$. Observe first that $v=\operatorname{Im}\{Q(\zeta)\}=\operatorname{Im}\{\zeta\}=\eta$, by the definitions of $Q$ and $F$. In other words, the mapping $Q$ sends each horizontal line into itself. In order to determine the action of $Q$ on a given horizontal line, we calculate the derivative

$$
\frac{\partial u}{\partial \xi}=\operatorname{Re}\left\{\frac{\partial}{\partial \xi} J_{\pi / 2}\left(J_{0}^{-1}(\zeta)\right)\right\}=\operatorname{Re}\left\{J_{\pi / 2}^{\prime}(z) / J_{0}^{\prime}(z)\right\}
$$


where $z=J_{0}^{-1}(\zeta)$. However, in view of the expressions for $H$ and $G$ in terms of $J_{\pi / 2}$ and $J_{0}$, we see that $J_{\pi / 2}^{\prime}=H^{\prime}+G^{\prime}$ and $J_{0}^{\prime}=H^{\prime}-G^{\prime}$. Thus

$$
\frac{\partial u}{\partial \xi}=\operatorname{Re}\left\{\frac{1+A(z)}{1-A(z)}\right\}>0, \quad z=J_{0}^{-1}(\zeta)
$$

because $|A(z)|<1$ in $D$. This implies in particular that $Q$ has a univalent restriction to each horizontal line that lies entirely in the horizontal slitdomain $J_{0}(D)$. Furthermore, $Q$ is univalent on each horizontal half-line or segment that ends at a boundary point of $J_{0}(D)$. To see that two such images cannot overlap, we have only to recall that $F$ sends each component of $\partial D$ to a point. The conclusion is that $Q$ is univalent on $J_{0}(D)$, performing a "horizontal shear" that collapses each boundary component to a single point. From the univalence of $Q$ it follows that $F$ is univalent in $D$, so it is a canonical punctured-plane mapping.

To prove the uniqueness, suppose that $\partial D$ has countably many components and let $F_{1}=H_{1}+\overline{G_{1}}$ and $F_{2}=H_{2}+\overline{G_{2}}$ be two canonical puncturedplane mappings of $D$, with $H_{k}$ and $G_{k}$ globally analytic in $D$. Since each $F_{k}$ $(k=1,2)$ is univalent and $F_{k}(\infty)=\infty$, the image $F_{k}(\partial D)=\partial F_{k}(D)$ is a bounded set of points. Thus it follows from the maximum principle that the harmonic function $f=F_{1}-F_{2}$ is bounded in $D$, because $f(\infty)=0$. Note also that $f$ is constant on each component of $\partial D$.

Consider now the analytic functions

$$
\Phi_{k, \alpha}=e^{i \alpha} H_{k}-e^{-i \alpha} G_{k}=e^{i \alpha} F_{k}-2 \operatorname{Re}\left\{e^{-i \alpha} G_{k}\right\}, \quad k=1,2
$$

and

$$
\psi_{\alpha}=\Phi_{1, \alpha}-\Phi_{2, \alpha}=e^{i \alpha} h-e^{-i \alpha} g=e^{i \alpha} f-2 \operatorname{Re}\left\{e^{-i \alpha} g\right\},
$$

where $f=h+\bar{g}$. Since each $F_{k}$ is univalent in $D$ and $\operatorname{Im}\left\{\Phi_{k, \alpha}(z)\right\}$ is constant on each component of $\partial D$, the argument principle shows that $\Phi_{k, \alpha}$ is univalent in $D$ for every $\alpha \in \mathbb{R}$. On the other hand, the normalization $F_{k}(z)=z+o(1)$ near infinity implies that $\Phi_{k, \alpha}(\infty)=\infty$. As a consequence, the two sets $\Phi_{k, \alpha}(\partial D)$ are bounded for each $\alpha$. Since $\psi_{\alpha}(\infty)=0$, we conclude from the maximum principle that $\psi_{\alpha}$ is bounded on $D$. However, we observe that $\operatorname{Im}\left\{\psi_{\alpha}\right\}=\operatorname{Im}\left\{e^{i \alpha} f\right\}$ is constant on each component of $\partial D$. Therefore, if $\psi_{\alpha}$ is not constant on $D$, then $\psi_{\alpha}(D)$ is a bounded domain whose boundary misses all but a countable number of horizontal lines, which is impossible. Thus $\psi_{\alpha}(z) \equiv 0$ in $D$ for every $\alpha$, since $\psi_{\alpha}(\infty)=0$. In other words, $g(z) \equiv e^{2 i \alpha} h(z)$ in $D$ for every $\alpha \in \mathbb{R}$. This shows that $h(z) \equiv g(z) \equiv 0$ in $D$, so $F_{1}=F_{2}$ and Theorem 3 is proved. 
The following remark may shed some light on the uniqueness assertion in the theorem. If an infinitely connected domain $D$ has a countable number of boundary components, then the canonical conformal mapping $J_{0}$ of $D$ onto a horizontal slit domain is uniquely determined. This is true more generally if $D$ admits a normalized conformal mapping onto a horizontal slit domain whose boundary components project orthogonally onto a set of measure zero on the imaginary axis. However, for an arbitrary domain containing infinity there may be more than one normalized conformal mapping onto a horizontal slit domain. (These results go back to Koebe [15]; see also Reich [23] and Kühnau [16].) Therefore, we cannot expect uniqueness of canonical harmonic punctured-plane mappings for arbitrary domains. Even in the case of nonuniqueness, however, it is possible to single out the (well-determined) mapping obtained through exhaustion by finitely connected domains. This will be called the canonical harmonic punctured-plane mapping of the infinitely connected domain.

The analytic function $G$ in the punctured-plane mapping $F=H+\bar{G}$ plays an important role in geometric function theory. Given a domain $D$ containing infinity, let

$$
\mathcal{D}(f, g)=\frac{1}{\pi} \iint_{D} f^{\prime}(z) \overline{g^{\prime}(z)} d x d y
$$

denote the Dirichlet integral of a pair of functions $f$ and $g$ analytic in $D$. Then $G$ has a basic "area-minimizing" property, as follows.

Theorem 4. Let $D$ be a domain containing infinity, not a punctured plane, and let $F=H+\bar{G}$ be the canonical harmonic punctured-plane mapping of $D$. Let $G$ have the expansion

$$
G(z)=\sum_{n=1}^{\infty} B_{n} z^{-n}
$$

near infinity. Then $B_{1}<0$ and $\mathcal{D}(f, f) \geq \mathcal{D}\left(G / B_{1}, G / B_{1}\right)$ for every function $f$ analytic in $D$ with the form

$$
f(z)=\frac{1}{z}+\sum_{n=2}^{\infty} c_{n} z^{-n}
$$

near infinity.

Proof. We will show that $\mathcal{D}(f, G)=-c_{1}$ for every analytic function $f$ with the form

$$
f(z)=\sum_{n=1}^{\infty} c_{n} z^{-n}
$$


near infinity. In particular, $-B_{1}=\mathcal{D}(G, G)>0$, or $B_{1}<0$. But the Schwarz inequality gives

$$
|\mathcal{D}(f, G)|^{2} \leq \mathcal{D}(f, f) \mathcal{D}(G, G),
$$

so it will follow that $\mathcal{D}(f, f) \geq-1 / B_{1}=\mathcal{D}\left(G / B_{1}, G / B_{1}\right)$ if $c_{1}=1$.

To prove that $\mathcal{D}(f, G)=-c_{1}$, it is enough to consider a finitely connected domain $D$ bounded by analytic Jordan curves; the general result will then follow by exhaustion. By the Cauchy-Green formula,

$$
\int_{\partial D} \bar{f} G^{\prime} d z=2 i \iint_{D} \overline{f^{\prime}} G^{\prime} d x d y
$$

where the boundary curves are traversed in the positive (clockwise) sense with respect to $D$. Thus

$$
\mathcal{D}(f, G)=-\frac{1}{2 \pi i} \int_{\partial D} f \overline{G^{\prime}} d \bar{z}
$$

On the other hand,

$$
H^{\prime} d z+\overline{G^{\prime}} d \bar{z}=d F=0
$$

on $\partial D$, because $F$ is a punctured-plane mapping. Consequently,

$$
\mathcal{D}(f, G)=\frac{1}{2 \pi i} \int_{\partial D} f H^{\prime} d z=-c_{1}
$$

by the residue theorem, since $H(z)=z+o(1)$ near infinity.

Corollary. If the domain $D$ omits a disk of radius $R$, then $\left|B_{1}\right| \geq R^{2}$, and this inequality is sharp.

Proof. Suppose for convenience that $D$ omits the disk $|z| \leq R$, and choose $f(z)=\frac{1}{z}$. Then

$$
\frac{1}{\left|B_{1}\right|} \leq \mathcal{D}(f, f)=\frac{1}{\pi} \iint_{D} \frac{1}{|z|^{4}} d x d y \leq \frac{1}{\pi} \int_{0}^{2 \pi} \int_{R}^{\infty} \frac{1}{r^{3}} d r d \theta=\frac{1}{R^{2}},
$$

or $\left|B_{1}\right| \geq R^{2}$. To see that the estimate is sharp, observe that $F(z)=z-R^{2} / \bar{z}$ is the canonical punctured-plane mapping of the domain $|z|>R$.

The following theorem was obtained in [11] for simply connected domains.

Theorem A. Let $a(z)$ be an analytic function on the unit disk $\mathbb{D}$ with $|a(z)|<1$. Let $\Omega$ be a bounded Jordan domain containing the point $w_{0}$. Then there exists a univalent solution of the Beltrami equation $\overline{f_{\bar{z}}}=a f_{z}$ 
such that $f(0)=w_{0}, f_{z}(0)>0, f(\mathbb{D}) \subset \Omega$ and $f^{*}\left(e^{i t}\right) \in \partial \Omega$ a.e., where $f^{*}$ denotes the radial limit of $f$.

Such a theorem does not hold on the domain $\Delta=\{z:|z|>1\}$ exterior to $\mathbb{D}$. Indeed, the following example was given in [12]. Let $a(z)=1 / z^{2}$ and let $K$ be a continuum. Then there is no univalent solution of $\overline{f_{\bar{z}}}=a f_{z}$ such that $f(z)=z+o(1)$ at infinity, $f(\Delta) \subset \Omega=\hat{\mathbb{C}} \backslash K$, and $f^{*}\left(e^{i t}\right) \in \partial \Omega$ a.e. In fact, the only univalent normalized solution is the canonical punctured-plane mapping $F(z)=z-1 / \bar{z}$.

We shall now see that this example is actually a special case of a much more general theorem. For a domain $D \subset \hat{\mathbb{C}}$ containing the point at infinity, let $\sum_{H}(D)$ denote the class of all univalent harmonic mappings $f$ defined on $D$ and normalized at infinity by $f(z)=z+o(1)$. Then in contrast to Theorem A, the following uniqueness theorem holds true.

Theorem 5. Let $D \subset \hat{\mathbb{C}}$ be a finitely connected domain containing the point at infinity, let $F=H+\bar{G}$ be the canonical harmonic punctured-plane mapping of $D$, and let $A=G^{\prime} / H^{\prime}$ be its dilatation function. Let $f \in \sum_{H}(D)$ be a solution of $\overline{f_{\bar{z}}}=A f_{z}$ in $D$. Then $f=F$.

Proof. With no loss generality, we may assume that each boundary component $C_{j}, j=1, \ldots, n$, is an analytic Jordan curve. Therefore, $F$ admits a harmonic continuation across $\partial D$ and $A$ has an analytic continuation across $\partial D$. Suppose first that $f$ is continuous together with its first partial derivatives in $\bar{D} \backslash\{\infty\}$. In what follows, integrations over the boundary $\partial D$ are taken in the positive (clockwise) direction with respect to $D$. By Green's theorem,

$$
\frac{1}{2 i} \int_{\partial D} \overline{f(z)} f^{\prime}(z) d z=-m(K) \leq 0,
$$

where $m(K)$ is the area of the compact set $K=\hat{\mathbb{C}} \backslash f(D)$ omitted by $f$. If $f \neq F$, then $F-f$ is a nonconstant orientation-preserving mapping, since it satisfies the Beltrami equation with $|A(z)|<1$ in $D$. Thus by the generalized argument principle (see [7]), $F-f$ is an open mapping. Since $F-f$ is smooth up to the boundary and $(F-f)(\infty)=0$, it follows that the area integral

$$
\begin{aligned}
& \iint_{D}\left\{\left|H^{\prime}-h^{\prime}\right|^{2}-\left|G^{\prime}-g^{\prime}\right|^{2}\right\} d x d y \\
& =\frac{1}{2 i} \int_{\partial D}\left\{(\bar{H}-\bar{h})\left(H^{\prime}-h^{\prime}\right)-(\bar{G}-\bar{g})\left(G^{\prime}-g^{\prime}\right)\right\} d z \\
& =\frac{1}{2 i} \int_{\partial D}(\bar{F}-\bar{f}) d(F-f)>0,
\end{aligned}
$$

where $f=h+\bar{g}$ and Cauchy's theorem for analytic functions has been applied. In other words, the last integral is finite and positive. On the other 
hand, $F$ is constant on each component of $\partial D$ and so $d F=0$. Consequently,

$$
\frac{1}{2 i} \int_{\partial D}(\bar{F}-\bar{f})(F-f)=\frac{1}{2 i} \int_{\partial D} \bar{f} d f-\frac{1}{2 i} \int_{\partial D} \bar{F} d f=-m(K) \leq 0
$$

a contradiction which proves that $f=F$ under the smoothness assumption. The result is now extended to the general case by applying the special result to a sequence of smoothly bounded domains $D_{n} \subset D$ which exhaust $D$. The conclusion is again that

$$
\iint_{D}\left\{\left|H^{\prime}-h^{\prime}\right|^{2}-\left|G^{\prime}-g^{\prime}\right|^{2}\right\} d x d y=0
$$

which implies that $f=F$. This proves the theorem.

Finally, the dilatation of the canonical harmonic punctured-plane mapping has a remarkable property: It maps the domain $D$ onto the $2 n$-times covered unit disk, where $n$ is the connectivity of $D$. This property was previously noted in [12] for doubly connected domains; see also [13]. The general result is essentially contained in Goluzin's book ([8], Chapters V and VI; see esp. pp. 291-292), where it is presented in a different context. Goluzin's parallel slit mappings are normalized at a finite point rather than at infinity, but small modifications of his argument establish this property of the dilatation.

Acknowledgments. The work of the first-named author was supported in part by an NSF grant (DMS-9401693), that of the second-named author by grants from NSERC and FCAR.

\section{References}

[1] L.V. Ahlfors, Conformal mapping, Lecture notes transcribed by R. Osserman, Dept. of Mathematics, Oklahoma A. and M. College, Stillwater, Oklahoma, 1951.

[2] D. Bshouty and W. Hengartner, Univalent solutions of the Dirichlet problem for ring domains, Complex Variables Theory Appl., 21 (1993), 159-169.

[3] Univalent harmonic mappings in the plane, Ann. Univ. Mariae CurieSkłodowska Sect. A, 47 (1994), 12-42.

[4] G. Choquet, Sur un type de transformation analytique généralisant la représentation conforme et définie au moyen de fonctions harmoniques, Bull. Sci. Math., 69 (1945), 156-165.

[5] J. Clunie and T. Sheil-Small, Harmonic univalent functions, Ann. Acad. Sci. Fenn. Ser. A.I, 9 (1984), 3-25.

[6] P. Duren and W. Hengartner, Harmonic mappings of multiply connected domains, Abstract No. 897-30-652, Abstracts Amer. Math. Soc., 16(1) (1995), 89.

[7] P. Duren, W. Hengartner and R.S. Laugesen, The argument principle for harmonic functions, Amer. Math. Monthly, 103 (1996), 411-415. 
[8] G.M. Goluzin, Geometric theory of functions of a complex variable, Moscow, 1952 (German transl., Deutscher Verlag, Berlin, 1957; 2nd ed., Izdat. Nauka, Moscow, 1966; English transl., Amer. Math. Soc., Providence, R.I., 1969).

[9] H. Grunsky, Lectures on theory of functions in multiply connected domains, Vandenhoeck \& Ruprecht, Göttingen, 1978.

[10] W. Hengartner and G. Schober, Univalent harmonic mappings onto parallel slit domains, Michigan Math. J., 32 (1985), 131-134.

[11] Harmonic mappings with given dilatation, J. London Math. Soc., 33 (1986), 473-483.

[12] Univalent harmonic exterior and ring mappings, J. Math. Anal. Appl., 156 (1991), 154-171.

[13] W. Hengartner and J. Szynal, Univalent harmonic ring mappings vanishing on the interior boundary, Canad. J. Math., 44 (1992), 308-323.

[14] H. Kneser, Lösung der Aufgabe 41, Jahresber. Deutsch. Math.-Verein., 35 (1926), 123-124.

[15] P. Koebe, Zur konformen Abbildung unendlich-vielfach zusammenhängender schlichter Bereiche auf Schlitzbereiche, Nachr. Akad. Wiss. Göttingen Math.-Phys. Kl., (1918), 60-71.

[16] R. Kühnau, Über ein Koebesches Beispiel zur Theorie der minimalen Schlitzbereiche, Wiss. Z. Martin-Luther-Univ. Halle-Wittenberg Math.-Natur. Reihe, 14 (1965), 319-321.

[17] R.S. Laugesen, Injectivity can fail for higher-dimensional harmonic extensions, Complex Variables Theory Appl., 28 (1996), 357-369.

[18] H. Lewy, On the non-vanishing of the Jacobian in certain one-to-one mappings, Bull. Amer. Math. Soc., 42 (1936), 689-692.

[19] A. Lyzzaik, Univalence criteria for harmonic mappings in multiply-connected domains, preprint, March 1995.

[20] Z. Nehari, Conformal mapping, New York, McGraw-Hill, 1952; Dover reprint, 1975.

[21] J.C.C. Nitsche, On the module of double-connected regions under harmonic mappings, Amer. Math. Monthly, 69 (1962), 781-782.

[22] T. Radó, Aufgabe 41, Jahresber. Deutsch. Math.-Verein., 35 (1926), 49.

[23] E. Reich, A counterexample of Koebe's for slit mappings, Proc. Amer. Math. Soc., 11 (1960), 970-975.

[24] N.M. Temme (editor), Nonlinear Analysis, Vol. 1, Mathematisch Centrum, Amsterdam, 1976 (Ch. II: "The topological degree of a mapping", 7-39).

Received October 4, 1995 and revised June 19, 1996.

\section{UNIVERSITY OF MiCHIGAN}

ANN ARBor, MichigAn 48109

E-mail address: duren@umich.edu

AND

UNIVERSITÉ LAVAL

QuÉBEC, P. Q. G1K 7P4

CANADA

E-mail address: walheng@mat.ulaval.ca 